\title{
Medialität und Interdiskursivität
}

\author{
ROLF PARR
}

\section{Medien, (Einzel-)Medium, Medialität}

Beschäftigt man sich mit Medialität, dann ist die heikelste Frage die nach einer Definition von Medium bzw. Medien, denn die inzwischen stark ausdifferenzierte medientheoretische Forschung ist sich, wenn überhaupt in irgendeinem Punkt, dann darin einig, dass ihre Überlegungen bisher noch nicht zu einem einheitlichen Verständnis des Begriffs >Medien< geführt haben. So stellen die in Umlauf befindlichen Definitionen jeweils ganz unterschiedliche Aspekte des Medialen in den Vordergrund: mal die technische Basis von Medien (von Pyramide über Buchdruck zu Telegrafenmast und Internet), mal ihre Funktion (Zeichenvorräte oder Kanäle für Kommunikation bereitstellen, Informationen speichern und damit über Zeit und Raum hinweg verfügbar machen, Interaktion in sozialen Teilsystemen ermöglichen), mal sind es aber auch gesellschaftliche Bezüge (wie etwa die Konstruktion kultureller Gegenstände oder die Manipulation der Mediennutzer), die favorisiert werden. ${ }^{1}$ Da die Medienwissenschaften aus so verschiedenen Bezugsfächern wie den Literatur-, Sprach- und Kulturwissenschaften, der Semiotik, Publizistik- und Kommunikationswissenschaft, Soziologie, Informationstheorie oder Kybernetik stammen, ist das auch nicht allzu verwunderlich. Eine Wesensbestimmung des Medialen schlechthin, also von >Medium < allgemein, ist dabei so wenig in Sicht, dass sich niemand ernsthaft an einer singulären Medientheorie mit vielleicht sogar historisch übergreifendem Geltungsanspruch abarbeitet. Deren Stelle nehmen bisher noch additiv verfahrende Überblicke ein, wie die von Werner Faulstich, ${ }^{2}$ Daniela Kloock und Angela Spahr ${ }^{3}$ oder Die-

1 | Vgl. Daniela Kloock/Angela Spahr: Medientheorien. Eine Einführung. 2., korr. u. erw. Aufl. München 2000, S. 8.

2 | Werner Faulstich: Medientheorien. Einführung und Überblick. Göttingen 1991. Ders. (Hg.): Grundwissen Medien. München 1998, S. 21-28 (Kap. "Medientheorie«).

3 | Ebd. 
ter Mersch, ${ }^{4}$ bzw. reine Textsammlungen zur Medientheorie. ${ }^{5}$ Nützlich scheint mir allerdings der Vorschlag von Jochen Schulte-Sasse zu sein, zunächst einmal eine >starke < und eine >schwache< Variante des Medienbegriffs voneinander zu unterscheiden:

Die schwache sieht das Medium als einen Informations- oder Kommunikationsträger, der auf das Übertragene nicht zwangsläufig einwirkt. Das Medium bleibt hier Instrument. [...] Die starke Bedeutungsvariante betrachtet das Medium als einen Träger von Informationen, der diese nicht mehr oder weniger neutral vermittelt, sondern sie grundsätzlich prägt, sich innen medienspezifisch einschreibt und dadurch dem menschlichen Zugriff auf Wirklichkeit Form verleiht. Den Medien wird hier eine nicht steuerbare, von inrer Form stärker als ihrem Inhalt beeinflußte Wirkung zugeschrieben [...]. ${ }^{6}$

Aber selbst mit dieser Aufteilung in zwei Gruppen von Medienbegriffen steht die Medientheorie, soweit sie nicht nur hochsektorielle Einzelmedientheorie ist, heute vor einem ganz ähnlichen Dilemma wie die Literaturwissenschaft Ende der $1960 e r$ Jahre, als sie einen Ausweg aus den bis dato stets gescheiterten Versuchen einer ontologischen Wesensbestimmung von Literatur suchte und die von Roman Jakobson entwickelte Vorstellung einer Vielzahl von genuin literarischen Verfahren aufnahm, die je nach Dichte abgestufte Grade von Literarizität zu beschreiben erlauben. Parallel zu diesem Literarizitätskonzept (und seiner unter den Stichworten >Theatralität< bzw. >Performativität< dann in der Theaterwissenschaft der frühen 199oer Jahre ein zweites Mal erfolgreichen Adaption7) könnte ein möglicher Weg auch für die Medientheorie darin liegen, zumindest probeweise nicht nur nach spezifischen Eigenschaften von (Einzel-)Medien, sondern auch nach spezifischen Verfahren von Medialität überhaupt zu fragen. Sie wären zwar unterhalb der Ebene umfassend ausformulierter Theorien angesiedelt, müssten aber dennoch so breit und sinnfällig im Material sichtbar sein, dass ihnen eine auch empirisch verifizierbare Signifikanz zukäme. Mit der Erforschung solcher durchgehenden Eigenschaften und Verfahren könnten dann zugleich Querstrukturen zwischen den einzelnen Medien und ihren Einzelmedientheorien sichtbar gemacht werden. Gegenüber dem Status quo medientheoretischer Reflexion wäre

4 | Dieter Mersch: Medientheorien zur Einführung. Hamburg 2006.

5 | Günter Helmes/Werner Köster (Hg.): Texte zur Medientheorie. Stuttgart 2002.

6 | Jochen Schulte-Sasse: Art. "Medien/medial«. In: Karlheinz Barck u.a. (Hg.): Ästhetische Grundbegriffe. Bd. 4. Stuttgart/Weimar 2002, S. 1-38, hier: S. 1.

7 | So erfolgreich, dass der Berliner Sonderforschungsbereich "Kulturen des Performativen " 2002 seine Jahrestagung unter das Thema "'performing media - Medien im Vollzug" stellte. Der Eröffnungsvortrag der Philosophin Sybille Krämer lautete dann entsprechend "Was hat ’Performativitäı mit ’Medialitätı zu tun?". Vgl. auch Sybille Krämer: Sprache - Stimme - Schrift: Sieben Gedanken über Performativität als Medialität. In: Uwe Wirth (Hg.): Performanz. Zwischen Sprachphilosophie und Kulturwissenschaften. Frankfurt a.M. 2002, S. 323-346. 
damit bereits eine ganze Menge gewonnen, denn die vorliegenden Theorien nehmen bisher - wie es in den einschlägigen Lexikon- und Handbuchartikeln ziemlich einhellig heißt - »in aller Regel [...] kaum aufeinander Bezug «. ${ }^{8}$

Als Parallelbegriff zu >Literarizität < würde >Medialität < dann die Menge der genuin medialen Eigenschaften und Verfahren meinen (siehe Schema 1). In diesem Sinne wird von Medialität beispielsweise von Knut Hickethier gesprochen, der den Begriff auf zwei Ebenen des Medialen bezieht: Erstens bezeichnet Medialität spezifische Eigenschaften, die »für alle Medien in gleicher Weise determinierend « sind, also »etwas Grundsätzliches, das die mediale Kommunikation insgesamt bestimmt«. Zu denken ist hier etwa an sämtliche Formen von Wiederholung, die technische Reproduzierbarkeit und damit Medialität voraussetzen. ${ }^{9}$ Zweitens »meint der Begriff das als typisch genommene Set von Eigenschaften, das für einzelne Medien als konstitutiv angesehen wird $«,{ }^{\circ}$ sodass nach einer spezifischen Medialität des Films (etwa der »technische[n] Wahrnehmungsanordnung des Kinos« und den filmischen »Produktions- und Distributionsformen«), des Radios (dem »Radiophonen«), des Fernsehens (dem »Audiovisuellen« bzw. noch spezifischer dem »Televisuellen«), der neuen »Netzmedien « usw. zu fragen wäre. ${ }^{11}$ Die medialen Eigenschaften auf beiden Ebenen werden dabei gleichermaßen durch die jeweils zugrunde liegende Technik hervorgebracht wie auch durch den kulturellen Gebrauch der Medien bestimmt. ${ }^{12}$

So weit könnte man die Begrifflichkeit für halbwegs aufgeräumt erachten, ${ }^{13}$ wenn in den Sprachwissenschaften und -didaktiken sowie der empirischen Bildungsforschung nicht seit der ersten PISA-Studie verstärkt von Literalität die Rede wäre, was nicht allein die spezifischen Eigenschaften geschriebener Sprache meint, sondern auch diejenigen Kompetenzen, über die Einzelindividuen verfügen müssen, um an schriftsprachlichen Kulturen sinnvoll teilhaben zu können.

8 | Faulstich, Medientheorien (Anm. 2), S. 23.

9 | Vgl. Rolf Parr: ,Wiederholen،. Ein Strukturelement von Film, Fernsehen und neuen Medien im Fokus der Medientheorien. In: kultuRRevolution. zeitschrift für angewandte diskurstheorie 47 (Juni 2004), S. 33-39.

10 | Knut Hickethier: Einführung in die Medienwissenschaft. Stuttgart/Weimar 2003, S. 26.

11 | Ebd., S. 357-359.

12 | Vgl. ebd., S. 27 u. 29.

13 | Unberücksichtigt bleibt hier die Verwendung des Begriffs für Personen (’Medien`) mit übernormalen Fähigkeiten, die als Instanzen der Vermittlung (des ımedialen Kontaktsı) zwischen Diesseitigem und Jenseitigem fungieren. 


\section{Schema 1: Kleine Synopse relevanter Begriffe}

\begin{tabular}{|c|c|c|}
\hline $\begin{array}{l}\text { Bezeichnung des } \\
\text { Gegenstandes }\end{array}$ & $\begin{array}{c}\text { Bezeichnung für die } \\
\text { spezifischen Verfahren bzw. } \\
\text { Eigenschaften }\end{array}$ & $\begin{array}{l}\text { Bezeichnung für die nötigen } \\
\text { Rezeptionskompetenzen }\end{array}$ \\
\hline Literatur & $\begin{array}{l}\text { Literarizität } \\
\text { (Roman Jakobsen; Begriff } \\
\text { zielt auf die Summe spezi- } \\
\text { fisch literarischer Verfah- } \\
\text { ren) } \\
\text { Literalität (vs. Oralität) } \\
\text { (Walter Ong; Begriff zielt auf } \\
\text { die Summe spezifischer Ei- } \\
\text { genschaften geschriebener } \\
\text { Spache) }\end{array}$ & $\begin{array}{l}\text { Literalität } \\
\text { (engl.: Reading-Literacy; Begriff } \\
\text { zielt auf die nötigen Kompeten- } \\
\text { zen der Rezipienten zur Teilhabe } \\
\text { an literalen Kulturen) }\end{array}$ \\
\hline Medien & $\begin{array}{l}\text { Medialiät } \\
\text { (Knut Hickethier; erstens } \\
\text { spezifische Eigenschaften, } \\
\text { die für alle Medien in glei- } \\
\text { cher Weise determinierend } \\
\text { sind; zweitens das für ein- } \\
\text { zelne Mediene als typisch } \\
\text { angesehene Set von Eigen- } \\
\text { schaften) }\end{array}$ & $\begin{array}{l}\text { [Medialität] oder Medienkompetenz } \\
\text { (engl.: Media-Literacy oder Digi- } \\
\text { tal-Literacy; Begriff zielt auf die } \\
\text { nötigen Kompetenzen der Rezi- } \\
\text { pienten zur Teilhabe an medialen } \\
\text { Kulturen) }\end{array}$ \\
\hline Einzelmedium Theater & Theatralität, Performativität &. \\
\hline Einzelmedium Fernsehen & Audiovisualität, Televisualität &.$\%$ \\
\hline Einzelmedium Film & Filmzitat, das Filmische & $\%$ \\
\hline
\end{tabular}

Gegenüber eher medientheoretischen Beschreibungen der Unterschiede zwischen Literalität und Oralität bei Walter J. Ong und anderen Literalitätsforschern ${ }^{14}$ bedeutet das eine Verschiebung hin auf die Rezipienten und ihre Kompetenzen. Für die Sprach- und Literaturwissenschaften ist das halbwegs unproblematisch, da man Literarizität von Literalität ja trotz der doppelten Besetzung von Literalität als Verfahren und als Rezeptionskompetenz noch recht gut voneinander unterscheiden kann. Schwieriger sieht es jedoch für die Medienwissenschaften aus, denn da heißt es für das Ensemble der typischen Verfahren ja nicht etwa >Mediarizität< oder >Medializität<, sondern nur Medialität, sodass man in Analogie zur Sprach- und Literaturwissenschaft denken könnte, Medialität sei für Medien das, was Literalität für die Rezeption von Schriftsprache sei, nämlich eine User-Kompetenz. Die Fähigkeiten der Rezipienten sind aber gerade nicht gemeint, wenn der Blick auf die >Medienhaftigkeit ' gelenkt werden soll und damit auf die Frage, wie durch verschiedene Medien verschiede-

14 | Walter J. Ong: Orality and Literacy. The Technologizing of the Word. London 1982 [dt.: Oralität und Literalität. Die Technologisierung des Wortes. Opladen 1987]. - Vgl. als 'Klassiker auch Jack Goody (Hg.): Literalität in traditionalen Gesellschaften. Frankfurt a.M. 1981. 
nes Wissen und verschiedene »Kulturen der Kommunikation « ${ }^{15}$ konstituiert werden. Nimmt man die englischen Begriffe hinzu, dann entspräche dem sprachwissenschaftlichen Literalitätsbegriff - englisch Literacy bzw. Reading-Literacy - derjenige der Media-Literacy bzw. eingeschränkter der Digital-Literacy oder im deutschsprachigen Raum der zwischen medienwissenschaftlicher und medienpädagogischer Akzentuierung changierende und daher enorm komplexe Begriff der Medienkompetenz, meist gebraucht im Sinne derjenigen Fähigkeiten und Fertigkeiten, über die Individuen oder Gruppen verfügen müssen, um »Massen- und Individualmedien zu handhaben, sich in der Medienwelt zurechtzufinden, Medieninhalte aufzunehmen, zu verarbeiten und gestalterisch in den Medienproduktions- und -distributionsprozeß eingreifen zu können «. ${ }^{16}$

Fasst man die bisherigen Überlegungen zusammen, dann kann man sagen, dass die »Beschäftigung mit Medien« nicht »von einer gesicherten Definition ihres Gegenstandes« ausgehen kann. Daraus hat das Forschungskolleg Medien und kulturelle Kommunikation an der Universität Köln die Konsequenz gezogen, die Frage nach dem Wesen des Medialen umzuformulieren in die sehr viel operativere Frage danach, wie Medialität funktioniert, ${ }^{17}$ wie sich »>Mediation < im Sinne von Vermittlung, Darstellung und Kommunikation ${ }^{18}$ in, mittels und zwischen den verschiedenen Medien als Prozess konkretisiert. Das ist vielfach auch das Ziel der Beiträge dieses Bandes, nämlich die mediale Konstitution, Transformation und Tradierung von Wissen in und durch ganz unterschiedliche Medien in den Blick zu nehmen und zu zeigen, wie diese nicht nur auf technisch differente Weise operieren, sondern auch verschiedenes Wissen modellieren, es auf ganz unterschiedliche Weise kommunizieren, in Umlauf bringen und schließlich ebenso differente Wahrnehmungsgewohnheiten etablieren, die synchron der Medienkonkurrenz, diachron dem Medienwandel unterliegen. Die meisten Beiträge setzen dementsprechend einzelne $\mathrm{Me}$ dien (wie Stimme, Körper, Reliquien, Pergament, Literatur, Buchdruck, Stummfilm oder neue Medien) mit mal historischen, mal aktuellen Themen (wie Nibelungen-Stoff, Dämonen und Vampire, Nation, Luxemburger Sprachraum, Frömmigkeit oder Shopping) und theoretischen Konzepten (wie Präsenz, Intermedialität, Medienkonkurrenz, Kybernetik, kollektives Gedächtnis oder symbolische Gewalt) in Verbindung. Die Fragestellung ist also vierteilig angelegt: Welche Medien konstituieren mit Bezug auf

15 | Kulturwissenschaftliches Forschungskolleg "Medien und kulturelle Kommunikation«. Forschungsprogramm online unter http://www.fk-427.de/Profil/Projekte [31.03. 2011].

16 | Klaus Neumann-Braun: Art. "Medienkompetenz". In: Alexander Roesler/Bernd Stiegler (Hg.): Grundbegriffe der Medientheorie. München 2005, S. 173-175, hier: S. 173.

17 | Kulturwissenschaftliches Forschungskolleg (Anm. 15).

18 | Mersch, Medientheorien (Anm. 4), Klappentext. 
welche Themen welches Wissen und unter Rückgriff auf welche Theoreme lässt sich das analysieren?

Demgegenüber soll das Problem der Medialität hier etwas anders angegangen werden und vom Ort der Interdiskurs-Theorie aus gefragt werden, ob es nicht auch solche materiell bestimmbaren und theoretisch beschreibbaren Phänomene von Medialität gibt, die quer dazu liegen, die sich also quer durch die Einzelmedien finden lassen, die zudem über ganz verschiedene Themen hinweg eine Rolle spielen und die möglicherweise auch noch für mehrere theoretische Zugriffe von Interesse sind bzw. mit diesen ein Stück weit kompatibel. Ein solches, weder einfach als >Hard-< noch als >Software< fassbares Phänomen scheint das der Interdiskursivität zu sein. Was Interdiskursivität mit Medialität zu tun hat und wie moderne Medien-Interdiskurse als ein Medium sui generis mit ganz eigener Medialität funktionieren, soll im Folgenden gezeigt werden.

\section{IINTERDISKURSIVITÄT`?}

Über so verschiedene theoretische Ansätze wie Niklas Luhmanns Systemtheorie, Reinhart Kosellecks historische Semantik, Michel Foucaults Diskurstheorie und eine ganze Reihe daran anschließender Kultur-, Literaturund Medientheorien hinweg hat sich als Konsens herausgebildet, dass moderne Gesellschaften etwa seit Beginn der zweiten Hälfte des 18. Jahrhunderts durch funktionale Ausdifferenzierung gekennzeichnet sind, das heißt durch die Entwicklung spezieller Praxis- und Wissensbereiche, die wiederum relativ geschlossene Spezialdiskurse ausgebildet haben, nämlich spezielle Formen der Rede mit eigener Operationalität. Demnach besteht die Gesamtkultur einer modernen Gesellschaft aus dem Spektrum ihrer Spezialdiskurse (z.B. naturwissenschaftlichen, human- und sozialwissenschaftlichen, kultur- und geisteswissenschaftlichen), aber auch aus Interdiskursen, die diese ausdifferenzierten Spezialdiskurse wieder re-integrieren. Denn würde es bei Spezialisierung allein bleiben, wäre Verständigung über die Grenzen der Spezialdiskurse hinweg heutzutage kaum mehr möglich. Genau das müssen viele Medien wie Presse, Rundfunk und Fernsehen aber leisten. Die modernen Gesellschaften haben sich daher nicht nur in arbeitsteilige Spezialbereiche ausdifferenziert, sondern als kompensatorische Antwort auf das immer weitere Auseinanderdriften der Spezialwissensbereiche auch solche diskursiven Verfahren entwickelt, die zwischen den Spezialisierungen wieder neue Verbindungen herstellen, also gleichsam Brücken schlagen; daher die Bezeichnung >Inter<-Diskurse. ${ }^{19}$ Dazu gehören beispielsweise alle Formen von Analo-

19 | Die einschlägige Forschungsliteratur verzeichnet: Rolf Parr/Matthias Thiele: Link(s). Eine Bibliographie zu den Konzepten "Interdiskurs", "Kollektivsymbolik" und "Normalismus" sowie einigen weiteren Fluchtlinien. Zweite, stark erw. u. überarb. Aufl., Heidelberg 2010. 
gien, Metaphern, Symbolen, Mythen und narrativen Stereotypen, wie sie bereits im Alltag (als einem solchen nicht-speziellen Lebensbereich) und dann gehäuft in Literatur und den verschiedenen (Massen-)Medien anzutreffen sind.

Einige noch recht einfach strukturierte Beispiele für solche Analogien aus einer einzigen Ausgabe der Financial Times Deutschland, einer Tageszeitung also, von der man eher auf Eindeutigkeit abzielendes, denotiertes Wirtschaftsspezialwissen als Interdiskurs-Elemente erwarten würde, können schnell deutlich machen, wie das >Ins-Spiel-Bringen < verschiedener gesellschaftlicher Teilbereiche allein schon in den Überschriften der Artikel funktioniert.

Abb. 1: Financial Times Deutschland (8. Februar 2007, S. 6)

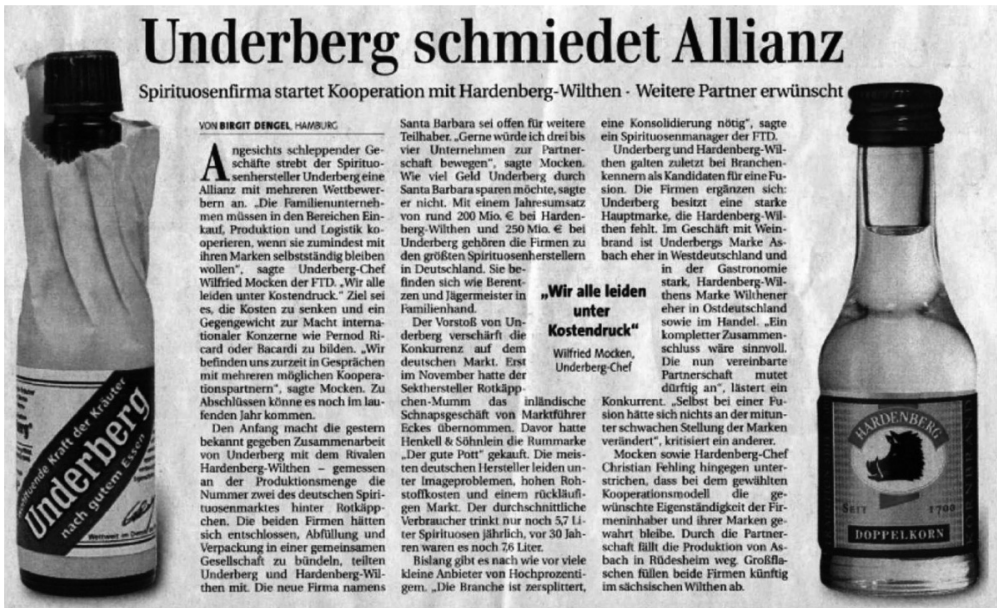

Abb. 2: Financial Times Deutschland (8. Februar 2007, S. 8)

\section{Roche bremst Euphorie der Anleger}

Schweizer Konzern stimmt Investoren auf wachstumsschwächere Zeiten ein. Gewinnerwartungen 2006 ủbertroffen

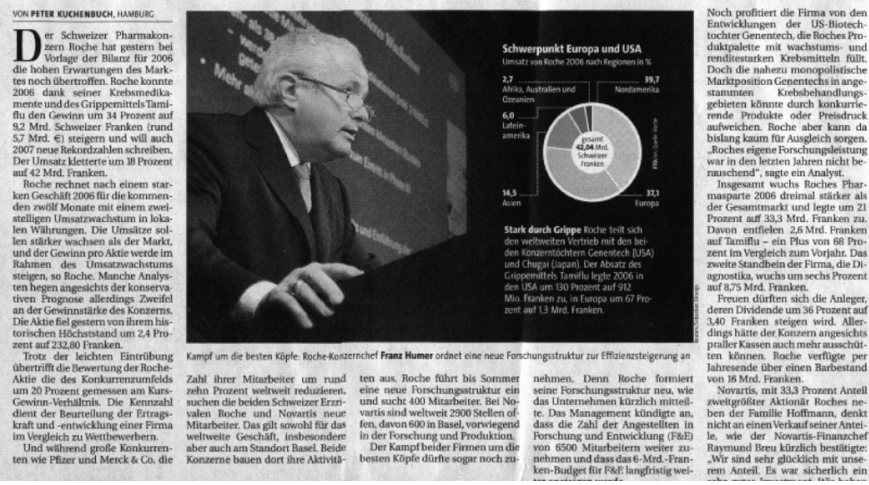


Abb. 3: Financial Times Deutschland (8. Februar 2007, S. 9)

\section{Kohlegipfel schafft den Durchbruch}

Bundesregierung und Kohleländer einigen sich auf Ausstieg aus der Förderung 2018 · Nordrhein-Westfalen erreicht Entlastung

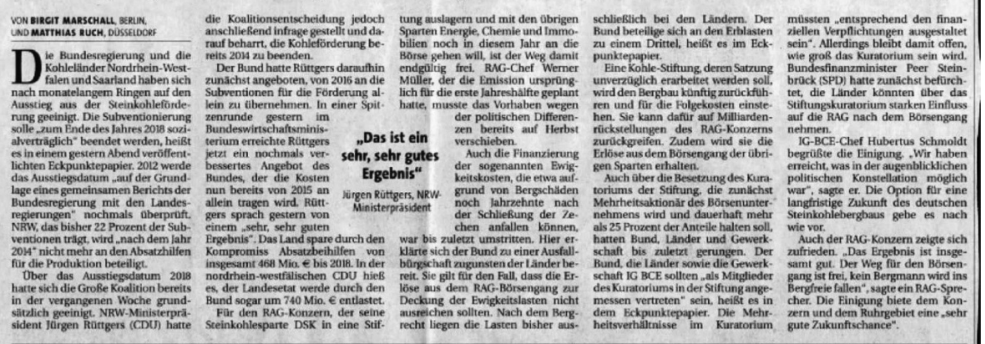

Abb. 4: Financial Times Deutschland (8. Februar 2007, S. 16)

\section{Deutsche Börse brütet über Strategie}

Unternehmen denkt über Finanzierung weiterer Aktienrückkäufe nach · Aufsichtsrat tagt am Montag außerordentlich

\begin{tabular}{|c|c|c|c|c|}
\hline & \multirow[b]{2}{*}{ 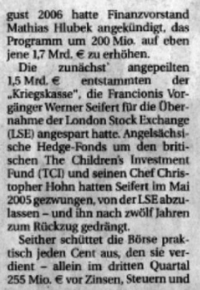 } & \multirow[b]{2}{*}{ 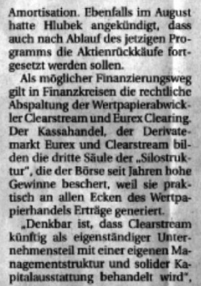 } & \multirow[b]{2}{*}{ 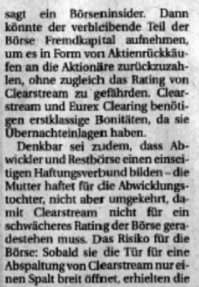 } & \multirow[b]{2}{*}{ 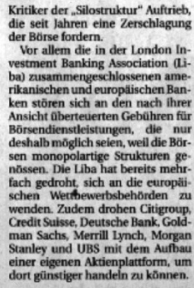 } \\
\hline & & & & \\
\hline
\end{tabular}


Abb. 5: Financial Times Deutschland (8. Februar 2007, S. 17)

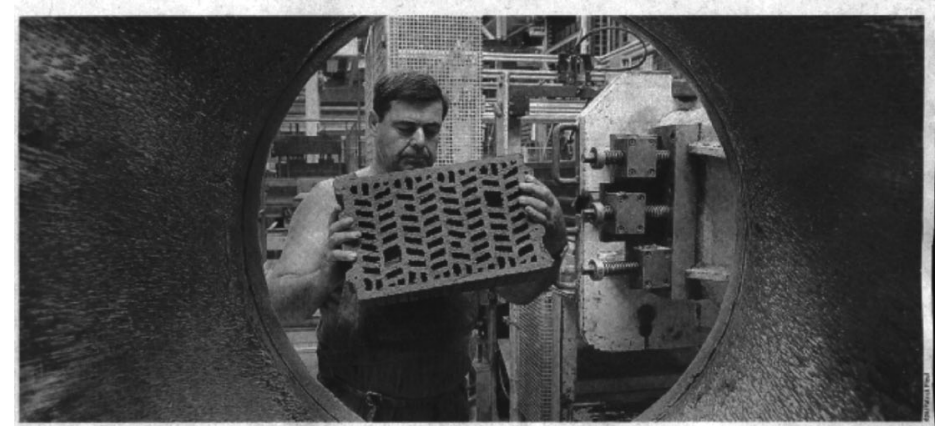

Nicht nur die Produhte des weltgrobiten Ziegelhesstellens Wienerberger sind gefragt. Auch um Anleihen des osterneichischen Unternehmens reilBeo sich die Anleger

\section{Firmen geben Bondmarkt einen Korb}

Emissionsflaute lässt Renditeaufschläge auf Rekordtief sinken · Investoren weichen auf Bankanleihen aus
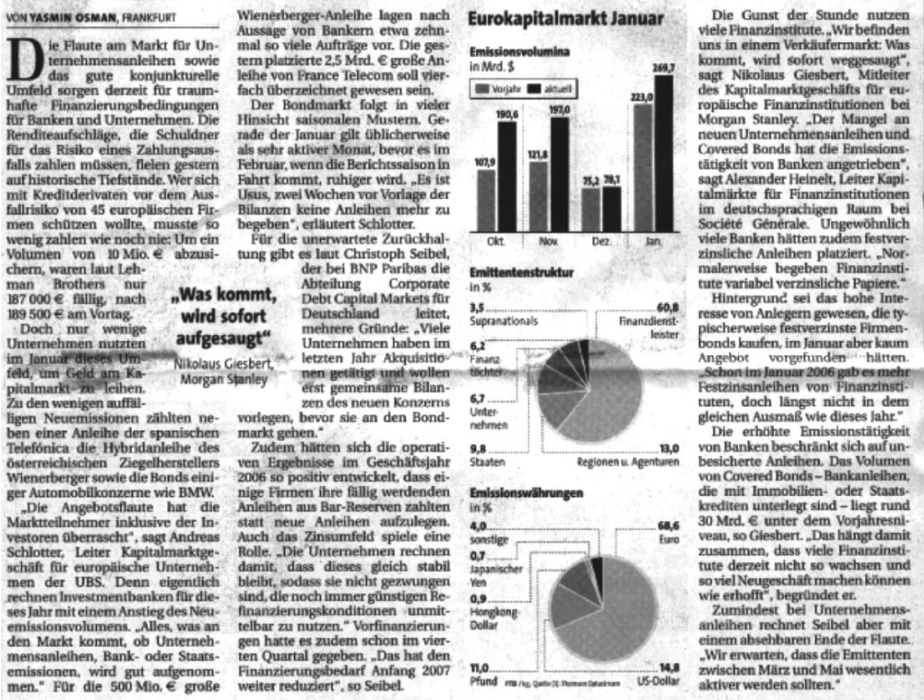
Abb. 6: Financial Times Deutschland (8. Februar 2007, S. 24)

\section{Transatlantisches Tauwetter}

Die Chancen für einen neuen Klimakonsens zwischen Europäern und Amerikanern stehen so gut wie nie

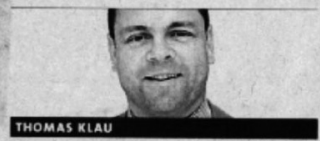

er globale Klimawandel ist nach aller-
grö̉ter Wahrscheinlichkeit das ErgebgröBter Wahrscheinlichkeit das ErgebFeststellung der Weltklimakonferenz Ende vergangener Woche in Paris hat in der amerikanischen Debatte - anders als in der europaisischen - eine echte Zasur markiert. Der Streit daruber, seit mehr als 20 Jahren politisch so gut wie abgeschlossen; auch konservative Partelen haben den Kampf gegen den Klimawandel liangst in ih
Programm ubernommen. In den Vereinigten Programm ubernommen. In den Vereinigten Staaien war das bislang anders.

Bis in die jangste Vergangenheit hinein fanden in den USA Wissenschaftler und Lobbyisten Gehör, die Warnungen vor einer Klimakatastrophe als apokalyptische Hysterie brandmarkten. Der Wetterwandel wurde geleugnet oder verharmlost als Resultat naturlicher Tem. lahthunderte und Jeh, wavsende im Laufe de ah. Militant konservative Publikationer sur. gab. Militant konservative Publikationen sug gerierten ihren Lesern, dass Europas Ansatz in
Sachen Klimaschutz ein Produkt typisch europaischer Zukunftsangst sei - womöglich verpaunden mit dem Wunsch, die USA irgendwie an Wachstum und Wohlstand zu hindern. An tieuropàismus in Form der Neigung, Europe Schwache und Perfidie zu unterstellen, ist in der US-Rechten mindestens ebenso fest verwurzelt wie der antiamerikanische Reflex in der europaischen Linken.

\section{Enstimmig gegen das Kioto-Protokoll}

Doch der amerikanische Widerstand gegen das Kioto-Protokoll war kein Monopol rechter Republikaner. In der europaischen Diskussion
wird oft vergessen, dass der Senat dem Kioto Vertrag vorgeshn Jahren einstimmig eine Kiotoerteilte, noch bevor der endgultige Vertrage entwurf vorlag, Dieser seltene Ausdruck politischer Gesctlossenheit machte jede Forderumg nach einer Ratifikation des Vertrags aussichtslos. Diese Tatsache wurde in Europa aus polit. scher Bequemlichkeit allzu leicht ignoriert

Die Debatte in den USA ist in den vergan-

genen Monaten in mancher Hinsicht an dem

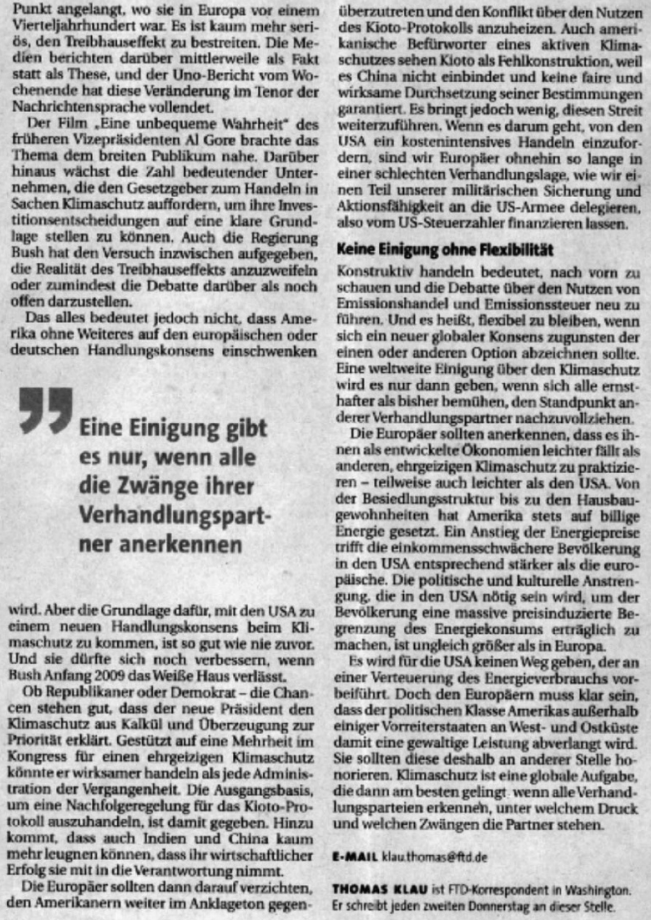

Solche auf Symbolisierung beruhenden Interdiskurs-Elemente bilden in ihrer Gesamtheit den allgemeinen Artikulationsrahmen eines Diskurssystems. Interdiskurse insgesamt stellen von daher eine Art Reservoir von Anschauungsformen für die notwendige Kodierung spezialdiskursiver Sachverhalte bereit, vor allem auch für diejenige aktueller Ereignisse (in den Beispielen u.a. für eine Wirtschaftskooperation, einen politischen Verhandlungserfolg über die Restlaufzeiten der Kohleförderung, für den Klimakonsens zwischen Europa und Amerika, das Anlageverhalten von Firmen und die Finanzierung von Aktien-Rückkäufen durch die Deutsche Börse AG). Rechnet man das, was hier nur einer Tageszeitungsausgabe entnommen ist, einmal hoch auf Rundfunk- und Fernsehsendungen, aber auch auf Kino, Werbung, Internet und Literatur, dann hat man es täglich mit einigen Dutzend, wenn nicht sogar einigen Hundert Symbolen in der Funktion von Interdiskurs-Elementen zu tun. Zu solchen nicht-speziellen, Kopplungen schaffenden Wissensbereichen gehören neben der Literatur als verbreitetem Interdiskurs auch philosophische, religiöse und weltanschauliche Interdiskurse und - als heute vielleicht wichtigster - der 
moderne Medien-Interdiskurs. Die Gesamtheit der diese Interdiskurse konstituierenden Verfahren ließe sich daher als die integrierende Kultur moderner Gesellschaften verstehen (das heißt so nebenbei liefert die Interdiskurs-Theorie auch noch eine originelle Definition von Kultur).

Der Medien-Interdiskurs, verstanden als das gesamte Ensemble der medial produzierten und distribuierten >Brückenschläge< zwischen Spezialwissensbereichen, ermöglicht es den Individuen, in hochgradig arbeitsteiligen und ausdifferenzierten Gesellschaften zu leben und zurecht zu kommen, ohne dabei ständig in verschiedenste Spezialisierungen und Professionalisierungen auseinandergerissen $\mathrm{zu}$ werden, das heißt Interdiskursivität »verwandelt die praktisch geteilte Arbeit imaginär in Lebenstotalität«, in Ganzheit. ${ }^{2 \circ}$ Dabei kann es natürlich nicht um vollständige Integration aller gesellschaftlichen Teilbereiche und aller menschlichen Fähigkeiten gehen, wie sie beispielsweise Friedrich Schiller in seinen Briefen Über die ästhetische Erziehung des Menschen ${ }^{21}$ entworfen hat und wie sie die Pädagogen des 19. Jahrhunderts dann für Schule (Stichwort >Allgemeinbildung<) und Universität (Stichwort >Studium generale <) vielfältig weitergesponnen haben, sondern nur um einzelne, in der Regel fragmentarisch bleibende >Brückenschläge <. Sie sind vor allem im Alltagswissen, in der Literatur, aber eben auch in den modernen Medien-Interdiskursen zu finden. ${ }^{22}$ Die Interdiskurs-Analyse versteht die modernen Medien-Interdiskurse daher als Orte, an denen sich solche verbindenden, der Re-Integration des in den Spezialdiskursen arbeitsteilig organisierten Wissens dienenden Elemente und Verfahren häufen. ${ }^{23}$ Sie bilden ein auf einer speziellen Form von Medialität beruhendes soziales Band der Integration.

An einem weiteren Beispiel kann man sich das besonders gut klar machen. Als die deutsche Bundeskanzlerin Angela Merkel Ende November 2006 beim Parteitag der CDU in Dresden eine Bilanz ihrer Politik vorlegen musste, da stand sie vor der Situation, ganz verschiedene Sachverhalte von Sozialpolitik über Globalisierung, Gesundheitsreform, Arbeitslosigkeit und Auslandseinsätze der Bundeswehr bis hin zu mangelndem

20 | Jürgen Link: Elementare Literatur und generative Diskursanalyse (mit einem Beitrag v. Jochen Hörisch u. Hans-Georg Pott). München 1983, S. 27.

21 | Friedrich Schiller: Über die ästhetische Erziehung des Menschen. In einer Reihe von Briefen. Mit e. Nachw. v. Käte Hamburger. Stuttgart 1977, bes. 19. bis 22. Brief, S. 75-92.

22 | Ich folge hier Jürgen Link: Zur Frage, was eine kulturwissenschaftliche Orientierung der Literaturdidaktik »bringen " könnte. In: kultuRRevolution. zeitschrift für angewandte diskurstheorie 45/46 (Mai 2003), S. 71-78.

23 | Vgl. Jürgen Link: Literaturanalyse als Interdiskursanalyse. Am Beispiel des Ursprungs literarischer Symbolik in der Kollektivsymbolik. In: Jürgen Fohrmann/Harro Müller (Hg.): Diskurstheorien und Literaturwissenschaft. Frankfurt a.M. 1988, S. $284-$ 307. Jürgen Link/Ursula Link-Heer: Diskurs/Interdiskurs und Literaturanalyse. In: LiLi. Zeitschrift für Literaturwissenschaft und Linguistik 20 (1990), H. 77, S. 88-99. 
Wirtschaftswachstum bündeln und zu ihrer Politik in Beziehung setzen zu müssen. Dazu hätte sie einen Bereich nach dem anderen mit Expertenwissen und in -sprache extensiv abhandeln können, was in sechs bis acht Stunden eventuell zu schaffen gewesen wäre. Das hätte aber erstens viel zu lange gedauert, zweitens hätten es nicht alle Zuhörer im Saal verstanden und drittens hätten die vielen Medienberichterstatter von Zeitungen, Rundfunksendern und nicht zuletzt Fernsehanstalten kein prägnantes und zitierfähiges Ergebnis in der Tasche gehabt, auf das sie in einer halben Spalte einer Zeitung, einer Sendeminute des Radios als O-Ton und einer halben Sendeminute des Fernsehens als O-Ton und -Bild hätten zurückgreifen können. Gerade das Fernsehen zielt aber »in besonders ausgeprägter Weise auf Reduktion von Komplexität und auf Anschlussfähigkeit an die elementare Soziokultur und die Alltagssprache«. ${ }^{24}$ Für eine Parteivorsitzende wie Angela Merkel wäre daher die Nicht-Anschlussfähigkeit der politische >Super-Gau< gewesen, nämlich über besonders wichtige Dinge zu reden, aber in der Öffentlichkeit damit überhaupt nicht wahrgenommen zu werden. In dieser Situation griff sie bzw. ihr Ghostwriter-Stab mit intuitiver Sicherheit auf das genuin interdiskursive Verfahren der Symbolisierung zurück, genauer gesagt auf ein kollektiv verbreitetes Symbol, das prinzipiell von jedermann/-frau verstanden und auch selbst produziert werden kann, nämlich das des Fußballs, und sagte gegen Ende ihrer Rede:

Nach der Weltmeisterschaft im eigenen Land, nach diesem einzigartigen Erlebnis der Gemeinschaft, des nationalen Zusammengehörigkeitsgefühls, der Freude und der Weltoffenheit sage ich es einfach in der Sprache des Fußballs: Ein Jahr nach der Bundestagswahl befinden wir uns in der 23. Minute eines Fußballspiels. Ja, wir haben schon einige tolle Tore geschossen. Ja, wir hatten einige gute Chancen, aber gewonnen ist noch gar nichts. Weitere 67 Minuten Spielzeit liegen vor uns. Es gibt viele weitere Möglichkeiten, Chancen für Deutschland herauszuholen und sie zu nutzen.

Wir müssen uns weiter anstrengen, nicht nur die ersten 23 Minuten zu gewinnen, sondern das ganze Spiel. Als Teamchefin habe ich dabei die Aufgabe, dass wir möglichst viele Chancen für Deutschland und für die Menschen in diesem Land nutzen. ${ }^{25}$

24 | Matthias Thiele: Flucht, Asyl und Einwanderung im Fernsehen. Konstanz 2005, S. 20 .

25 | Rede der Vorsitzenden der Christlich Demokratischen Union Deutschlands, Bundeskanzlerin Dr. Angela Merkel MdB. Auszug aus dem Stenografischen Protokoll. Dresden, 27. November 2006. 20. Parteitag der CDU Deutschlands, online unter http://www.dresden2006.cdu.de/download/061127_parteitag_rede_merkel.pdf [31.03.2011]. - Zur Fußball-Symbolik im Vorfeld der Weltmeisterschaft 2006 vgl. Rolf Parr: Anpfiff für spannende 90 Minuten Unterricht. Die Kollektivsymbolik des Fußballs. In: Praxis Deutsch 196 (März 2006), S. 45-51. 
Genau das war dann auch die Passage der insgesamt 27 Druckseiten umfassenden Rede, die in den Medien wieder aufgenommen und kommentiert wurde, sodass das Ziel medialer Wahrnehmung erreicht war.

\section{Kollektivsymbole und ihre Systeme}

Kollektivsymbole wie das bei der Parteitagsrede verwendete des Fußballs stellen diskurstheoretisch betrachtet Kopplungen von Spezialdiskursen und -wissensbereichen dar, hier konkret von >Sport< und >Politik<. Zeichentheoretisch betrachtet sind es komplexe, ikonisch motivierte und paradigmatisch expandierte Zeichen, die eine Bildseite (Pictura) und eine Seite des eigentlich Gemeinten (Subscriptio, >Sinn $<$ ) vereinen. ${ }^{26}$ Von daher lassen sie sich am einfachsten mit Hilfe eines Zwei-Kolonnen-Schemas analysieren, in das man fortlaufend zunächst einmal alle im Text realisierten Bild- und Sinn-Elemente einträgt und dann die fehlenden >SinnElemente< ergänzt (hier durch eckige Klammern markiert). Das sieht für das Beispiel umgesetzt so aus:

\section{Schema 2: Zwei-Kolonnen-Schema}

Pictura

$\mathrm{P}_{1}$ : $\quad$ wir befinden uns in der 23. Minute eines Fußballspiels

$\mathrm{P}_{2}$ : wir haben schon tolle Tore geschossen

$\mathrm{P}_{3}$ : wir hatten einige gute Chancen
Subscriptio

$\mathrm{S}_{1}: \quad$ ein Jahr nach der Bundestagswahl

$\mathrm{S}_{2 \mathrm{a}}$ : [wir haben einige unserer politischen Vorhaben durchgesetzt]

$\mathrm{S}_{2 \mathrm{~b}}$ : [wir haben Deutschland weiter gebracht]

$\mathrm{S}_{2 \mathrm{c}}$ : [wir haben der Opposition Abstimmungsniederlagen beigebracht]

$\mathrm{S}_{3}: \quad$ [politische Möglichkeiten]

26 | Die Terminologie wird entwickelt bei Jürgen Link: Die Struktur des Symbols in der Sprache des Journalismus. Zum Verhältnis literarischer und pragmatischer Symbole. München 1978. - Ein Verortung der Kollektivsymbolanalyse im Feld der Bildlichkeitsforschung und zugleich einen historischen Überblick bieten Axel Drews/Ute Gerhard/ Jürgen Link: Moderne Kollektivsymbolik. Eine diskurstheoretisch orientierte Einführung mit Auswahlbibliographie (Teil I). In: Internationales Archiv für Sozialgeschichte der deutschen Literatur. 1. Sonderh. Forschungsreferate (1985), S. 256-375. Frank Bekker/Ute Gerhard/Jürgen Link: Moderne Kollektivsymbolik. Ein diskurstheoretisch orientierter Forschungsbericht mit Auswahlbibliographie (Teil II). In: Internationales Archiv für Sozialgeschichte der Literatur 22 (1997), H. 1, S. 70-154. 
$\mathrm{P}_{4}: \quad$ gewonnen ist noch gar nichts

$P_{5}: \quad 67$ Minuten Spielzeit liegen $S_{5}$ : noch vor uns

$\mathrm{P}_{6}$ : viele weitere Möglichkeiten, $\mathrm{S}_{6}$ : Chancen für Deutschland herauszuholen

$\mathrm{P}_{7}$ : nicht nur die ersten $23 \mathrm{Mi}-$ nuten gewinnen, sondern das ganze Spiel

$\mathrm{P}_{8}$ : Teamchefin

\section{$\mathrm{P}_{9:} \quad$ Chancen für Deutschland} nutzen
$\mathrm{S}_{4}: \quad$ [es ist noch nicht sicher, dass wir das nächste Mal wiedergewählt werden]

[noch drei Jahre bis zur nächsten Wahl]

[weitere politische Vorhaben verwirklichen]

$\mathrm{S}_{7}: \quad$ [weiter machen Leute!]

$\mathrm{S}_{8 \mathrm{a}}: \quad$ [Parteivorsitzende Merkel]

$\mathrm{S}_{8 \mathrm{~b}}: \quad$ [Bundeskanzlerin Merkel]

$\mathrm{S}_{9}: \quad$ [Politik wirklich umsetzen]

Bereits an diesem noch sehr einfach strukturierten Beispiel lassen sich einige Definitionsmerkmale von Kollektivsymbolen festmachen:

- Erstens sind sie offensichtlich zweigliedrig aufgebaut. Sie besitzen eine Bildseite (Pictura) und eine des >eigentlich Gemeinten<, des Sinns (Subscriptio).

- Zweitens besteht die Bildseite aus mehreren zusammengehörigen Teil-Bildern, die sogar komplexere Text-/Bild-Korrelate fortlaufend strukturieren können. >Horizontal< gesehen ist also ein Pictura- jeweils einem Subscriptio-Element zugeordnet, während die beiden Reihen >vertikal< zu einer zumindest rudimentären Isotopie expandiert sein müssen. Das unterscheidet Kollektivsymbole von klassischen Ein- bzw. Zweiwort-Metaphern.

- Drittens lässt sich die Beziehung zwischen Pictura und Subscriptio näher bestimmen. Sie ist nicht völlig willkürlich, sondern semantisch motiviert. Eine Niederlage hätte man nicht so gut nutzen können.

- Viertens erfüllen Kollektivsymbole das Kriterium der Ikonizität, das heißt die Pictura-Elemente können bildlich dargestellt werden. Eine einfache Probe darauf, ob man es mit einem Kollektivsymbol zu tun hat, ist daher die Frage, ob sich ein entsprechender Text in eine Karikatur überführen lässt.

- Fünftes Merkmal ist die Tendenz zur Polysemie, zur Mehrdeutigkeit, das heißt unter einem Bild können durchaus verschiedene sinnvolle >Bedeutungen < gebildet werden. So lassen sich im Beispieltext für das Pictura-Element $\mathrm{P}_{2}$ (»wir haben schon tolle Tore geschossen «) die Subscriptio-Elemente $\mathrm{S}_{2 \mathrm{a}}$ bis $\mathrm{S}_{2 \mathrm{c}}$ gleichermaßen sinnvoll bilden (ebenso sieht es bei $\mathrm{S}_{8 \mathrm{a}}$ und $\mathrm{S}_{8 \mathrm{~b}}$ aus). Es ist diese 
Mehrdeutigkeit von Symbolen, die oftmals auch diejenige ganzer Texte und erst recht komplexerer Text-/Bild-Korrelate ausmacht, was emphatisch (und ebenfalls symbolisch) dann meist >Tiefe genannt wird.

Der Medien-Interdiskurs produziert nun solche Kollektivsymbole nicht ständig neu, sondern es lassen sich einige Dutzend relativ stabile, immer wiederkehrende Symboliken empirisch identifizieren, z.B. >Organismus <, >Körper<, >Schiff<, >Auto<, >Deich/Flut<, >Sport< usw., Symbole, die zwar mit verschiedenen Spezialdiskursen verbunden sein können (so z.B. >Organismus < und >Körper < mit der medizinischen Wissenschaft), die aber jenseits solcher Spezialität in ganz verschiedenen Diskursen vorkommen und zugleich durch ganz unterschiedliche soziale Träger verwendet werden können. So wird der politische Berichterstatter vielleicht vom >Abstieg Deutschlands in die zweite Liga < sprechen und schon nach einem neuen >politischen Nationaltrainer< Ausschau halten, der Wirtschaftsjournalist vom >immer noch stotternden motor , der Kulturphilosoph vom >kranken Patienten Deutschland s sprechen und die Bundeskanzlerin sich eben die gute Fußballstimmung des Sommers 2006 zu Nutze machen.

In ihrer Gesamtheit bilden solche Kollektivsymbole ein sich historisch zwar modifizierendes, synchron jedoch relativ stabiles und in sich kohärentes System. Dieser Systemcharakter resultiert daraus, dass Kollektivsymbole sowohl auf Seiten der Pictura als auch der Subscriptio zu paradigmatischen Äquivalenzklassen tendieren, das heißt sich wechselseitig ersetzen können. Zum einen können Pictura-Elemente (Bildlichkeiten) aus verschiedenen gesellschaftlichen Bereichen bei gleich bleibendem >Sinn< untereinander ausgetauscht werden. So lässt sich ein Gesellschaftssystem mal als Fahrzeug (z.B. Auto, Boot, Flugzeug, Zug oder Fahrrad) symbolisieren, dann aber auch als Organismus (mit Kopf, Herz, den verschiedenen Gliedmaßen, dem Blutkreislauf usw.). Daraus ergeben sich Ketten von verschiedenen Bildern bei gleich bleibendem >Sinn $<$, etwa: $>$ Politik $<$ ist wie >Fußball<, ist wie die >Regiearbeit an einer Oper $<$, ist wie der >Kopf $<$ des Volks->körpers $<$. Nimmt man etwa die Situation einer Vorlesung zum Thema Medialität, dann könnte man genauso gut sagen >wir sitzen alle zusammen im Boot der Medialitätsfrage<, wie auch >wir stricken alle zusammen am Pullover der Medialität< oder > wir bauen alle zusammen am Haus der Medialität<. Zum anderen können nun aber auch verschiedene Sachverhalte unter einem Bild subsumiert werden. >Flut<Symbole stehen z.B. gleichzeitig sowohl für Wassermassen, Flüchtlinge und Fußballfans wie auch Autokolonnen bei Beginn der Sommerferien; >Fundamentalisten < können grün, islamisch, serbisch oder orthodox sein. Aus diesen beiden Strukturachsen resultiert insgesamt der Charakter der Kollektivsymbolik als ein komplexes, synchrones System, das zwar aus vielen einzelnen, etwa 100 bis 150 relevanten Symbolen besteht, die aber untereinander in Beziehung gesetzt sind und immer wieder herangezo- 
gen werden, um Ereignisse jeglicher Art in der öffentlich-medialen Darstellung zu kodieren.

Sehr viel öfter als das Sprechen und Schreiben in einer einzigen kohärent durchgehaltenen Symbolik ist in Presse, Rundfunk und Fernsehen jedoch der fortlaufende Bildbruch (Katachresenmäander) anzutreffen, der den eigentlichen Normalfall des integrierenden >Ins-Spiel-Bringens verschiedener gesellschaftlicher Teilbereiche darstellt. Hier ein besonders instruktives Beispiel aus dem Wirtschaftsteil der Westdeutschen Allgemeinen Zeitung:

Wenn die Preise ins Kraut schießen, müssen sie einen Dämpfer erhalten. Und wenn dem Konjunkturmotor Überhitzung droht, muss frisches Kühlwasser her. Zur Abwehr der Inflationsgefahren sind die Hüter der Preisstabilität aufgerufen, ihre Instrumente einzusetzen. Gestern hat die Europäische Zentralbank (EZB) gehandelt. Und die Leitzinsen für den Euro erhöht.

Doch an der Preisfront lodern gar keine Feuer. Es gab nichts zu löschen. Und so bestand auch kein Anlass, wegen gestiegener Preise die geldpolitischen Zügel zu straffen.

Es ist wohl eher der Sturzflug des Euro, der die EBZ an der Zinsschraube drehen ließ. Sozusagen in der Rolle als Währungshüter. [...]

Auch nach der Erhöhung der Leitzinsen ist kein Ende der Talfahrt abzusehen. [...] Der schwache Eurokurs wird von einigen Politikern und Wirtschaftsfachleuten sogar insgeheim begrüßt. Denn ohne Zweifel wirkt jeder Euro-Cent, den die europäische Währung gegenüber dem Dollar und dem englischen Pfund verliert, wie eine Konjunkturspritze zugunsten der europäischen Exportwirtschaft. ${ }^{27}$

Ähnliches bietet jeden Tag die Kürzestsendung Börse im Ersten in der ARD vor der Tagesschau. Dieses Fernsehformat versucht, einen Wust von tagesstatistischen Entwicklungen, FirmenNachrichten, weltpolitischen Vorkommnissen und Börsenergebnissen in eineinhalb bis höchstens dreieinhalb Minuten anschaulich auf den Punkt zu bringen und greift dabei mit traumwandlerischer Sicherheit auf solche Mäander von ineinandergeschachtelten Kollektivsymbolen zurück, die Spezialwissen anschaulich machen und zugleich nahezu das gesamte Spektrum der Spezial- und Interdiskurse der bundesdeutschen Kultur ins Spiel bringen. Hier ein exemplarischer Textausschnitt aus der Sendung vom 11. Juli 2005, in der mehr als 30 Kollektivsymbole und Metaphern (kursiv gekennzeichnet) auf allerengstem Raum miteinander verschnitten wurden: ${ }^{28}$

Guten Abend! Zeichnungsgewinne?, werden sie vielleicht sagen - nie gehört. Stimmt nicht! Vor fünf Jahren war das noch das Einzige, worauf die Anleger scharf waren. Jetzt

27 | Lothar Noll: Zins hilft nicht. In: Westdeutsche Allgemeine Zeitung v. 28.04.2000, S. 2 (Hervorh. d. Verf.).

28 | Vgl. dazu Rolf Parr: Börse im Ersten: Kollektivsymbole im Schnittpunkt multimodaler und multikodaler Zeichenkomplexe. In: Mitteilungen des Deutschen Germanistenverbandes 54 (2007), H. 1 ("Medialitiät und Sprache»), S. 54-70. 
sind wieder viele freudig erregt. Teflon-Börse nennt man sie, weil viele schlechte Nachrichten an ihr einfach abtropfen. Spaß muss sein. Der Dax auf Jahreshoch, plus 65, so stark war das Aktienbarometer seit drei Jahren nicht mehr. Vorne ein Sensibelchen, Infinion, Speicherchips werden teurer, schön für die Münchener. Auch Autos fahren vor. VW profitiert von der Hoffnung, dass in dem Laden jetzt uffgeräumt wird. Jubel um Mobilcom und Freenet, beide wollen gemeinsam wachsen, na denn. Die Anschläge von London - sofort wieder verdaut. Die wirtschaftlichen Schäden gering, allein das ist, was zählt. Und noch gibt's Schwung auf der Orgel, wegen der Bundestagswahl.

Den modernen Medien-Interdiskursen, wie sie uns das Fernsehbeispiel in kondensierter Form vorgeführt hat, kommt aus interdiskurs-theoretischer Sicht ein quasi paradoxer Status zu: Einerseits sind sie als Spezialdiskurse zu beschreiben, da sie eigenen Formationsregeln unterliegen (z.B. dem tendenziellen Gesetz der ästhetischen Innovation im Falle der Literatur und dem der Aktualität im Falle von Fernsehen und Presse). Andererseits greifen sie, da sie kein eigenes genuines Thema haben, in besonders hohem Maße auf diskursübergreifende Elemente zurück und bringen im Extremfall das gesamte Spektrum der Spezial- und Interdiskurse einer Kultur ins Spiel. Eine interdiskurs-theoretisch orientierte Medienanalyse stellt daher nicht nur einen ausgesprochen operationalen Zugriff bereit, sondern ist zudem in der Lage, die verschiedenen Dimensionen des Medialen, im Falle des Fernsehens etwa Bild, Ton, Text, Programm, Serialität usw. einzubeziehen. Insgesamt wäre der moderne Medien-Interdiskurs dann als ein Spezialdiskurs zu beschreiben, dessen vorrangige Aufgabe darin besteht, nicht-spezialistische, interdiskursive Elemente und als deren kohärente Vernetzung ganze Interdiskurse zu produzieren. >Kultur wäre analog dazu als das durch Interdiskurse immer wieder neu integrierte Ensemble ausdifferenzierter moderner Wissensbereiche zu verstehen und >elementare Kultur< als das Integralwissen des Alltags.

Zusammenfassend kann man sagen: Interdiskurse generell und insbesondere der moderne Medien-Interdiskurs mit seinem System der Kollektivsymbole »sind die Lösungsfunktion für das Grundproblem hochdifferenzierter Gesellschaften: Sie decken den zunehmenden Bedarf an Kopplungen zwischen « den - in der systemtheoretischen Terminologie Niklas Luhmanns gesprochen - »einzelnen Teilsystemen sowie zwischen den funktionsspezifischen Kodes und den >interpenetrierenden < [...] psychischen Systemen «. ${ }^{29}$ Die Interdiskurs-Theorie selbst stellt ein theoretisches Modell für die Beschreibung des Prozesses der kulturellen Zusammenführung von Wissensbeständen über ein Ensemble von im weitesten Sinne analogiebildenden Verfahren bereit. Damit gibt sie zugleich eine Antwort auf die Frage nach dem Funktionszusammenhang von Alltag, Mediendiskursen, Spezialdiskursen und Kultur einer Gesellschaft.

29 | Benno Wagner: Im Dickicht der politischen Kultur. Parlamentarismus, Alternativen und Mediensymbolik vom "Deutschen Herbst" bis zur "Wende". München 1992 [= Materialität der Zeichen A 8], S. 341. 


\section{Medium InterdiskuRs?}

Da Kollektivsymbole und Interdiskurse insgesamt eine vermittelnde Funktion erfüllen, Themen und soziale Gegenstände sowie das Wissen über sie mit konstituieren, stellen sie der Fragestellung des Bandes entsprechend ein Medium sui generis mit eigener Spezifik, sprich eigener Medialität dar, die quer durch die Einzelmedien anzutreffen ist. Macht man sich beispielsweise noch einmal klar, dass für die sogenannten Massenmedien Zeitung, Radio und Fernsehen gleichermaßen das strikte Gebot der Platz- bzw. Zeitökonomie gilt (man hat nur eine halbe Spalte an Text oder nur eine Sendeminute zur Verfügung), dann können alle diese Medien das Problem im Rückgriff auf solche Interdiskurs-Elemente wie Kollektivsymbole lösen. Und sie werden es tun, wie die Beispiele gezeigt haben. Die spezifische Medialität von Zeitung, Radio und Fernsehen geht also in diesem Punkt Hand in Hand mit der Vermittlungsleistung von Interdiskursivität. Wenn Medientheoretiker immer wieder festgestellt haben, dass Medien ihre Existenz einem Problem der Abwesenheit verdanken, und daher eine Distanz oder Differenz überbrücken müssen, dann leisten Interdiskurse dies in ganz spezifischer Weise und in besonderem Maße. ${ }^{30}$

Auch das, was anscheinend die spezifische Medialität eines Einzelmediums ausmacht, ist häufig interdiskursiv basiert. Nimmt man etwa als mediale Besonderheit des Fernsehens den Anschluss an die elementare Sozialkultur (symbolisch den >kleinen Mann auf der Straße <), dann zieht dies fast automatisch interdiskursive Formen der Äußerung und der Darstellung nach sich. Der häufigste von Moderatoren an sogenannte Experten gerichtete Satz ist wahrscheinlich: >Können Sie das auch einfacher sagen?< Schließlich kommen überall da, wo man es mit hybriden Mischformen aus Text und Ton (z.B. Rundfunk) bzw. Text, Bild und Ton (z.B. Fernsehen) zu tun hat, häufig auch Kollektivsymbole ins Spiel, die auf diesen zwei bzw. drei Ebenen arbeitsteilig durchgespielt werden. So kann im Fernsehen beispielsweise die Subscriptio zu einer per Text eingebrachten Symbolik via Bild geliefert werden, wobei alle nur denkbaren Verteilungen von Pictura und Subscriptio auf Text, Bild und Ton denkbar sind. ${ }^{31}$ Damit wird ein spezifisches mediales Potential des Fernsehens in Verbindung mit der Medialität von Kollektivsymbolen als InterdiskursElementen deutlich, das über die eingeschränkteren Möglichkeiten allein schriftsprachlicher oder allein ikonografischer Präsentation hinausgeht. Das Fernsehen kann nämlich Pictura und Subscriptio nicht nur auf verschiedene Kodes verteilen, sondern das sowohl nacheinander als auch simultan. Das gilt ähnlich auch für das Verhältnis von Text und Bild bei Internetseiten, die ebenfalls sehr häufig im Verhältnis von Pictura und

30 | Vgl. dazu Jochen Hörisch: Der Sinn und die Sinne. Eine Geschichte der Medien. Frankfurt a.M. 2001, S. 34.

31 | Vgl. dazu Parr, Börse im Ersten (Anm. 28). 
Subscriptio zueinander stehen. Gegenüber dem Fernsehen eröffnet die Hypertextstruktur dabei die zusätzliche Möglichkeit, die Subscriptio >hinter die auf erster Ebene realisierte Pictura zu legen (und umgekehrt), sodass man durch Anklicken zwischen den beiden Seiten des Symbols wechseln kann.

Interdiskursivität spielt weiter auch für die Frage nach Intertextualität und Intermedialität eine wichtige Rolle. Denn fragt man genauer nach, worin das >inter < wirklich materialiter besteht, dann wird man sehr schnell sehen, dass Intertextualität bzw. Intermedialität als das, was materiell dann wirklich gemeinsam ist, Interdiskursivität immer schon voraussetzt. ${ }^{32}$ Das gilt auch für rein literarische Formen von Intertextualität. »Avancierte Intermedialitätskonzepte«, so Uwe Wirth, »fassen die mediale Transformation « als eine immer hybride bleibende Form der Verschmelzung auf, bei der die Differenz zwischen den beteiligten Medien ein Stück weit erhalten bleibt. ${ }^{33}$ Das trifft aber auch für Interdiskursivität zu: Sie lässt sich trotz aller Brückenschläge und Kopplungen immer noch auf die in sie eingehenden Spezialdiskurse zurückführen (das haben etwa die Beispiele aus der Financial Times Deutschland klar gemacht), stellt aber als ein auf Nicht-Spezialität spezialisierter Diskurs zugleich etwas Eigenes dar.

Eine spezifische Form von >Medium < bilden Kollektivsymbole und andere Interdiskurs-Elemente schließlich auch insofern, als sie einen nicht zu unterschätzenden Faktor für das kollektive Gedächtnis von Kulturen darstellen. Die Teilnehmer des merkelschen >Fußball<-Parteitags werden sich nach einiger Zeit kaum noch an die speziellen Fachinformationen erinnern, mit hoher Wahrscheinlichkeit aber an die fußballerische SchlussSymbolik, und sie werden sie womöglich aufgreifen und in abgewandelter Form selbst wieder anwenden. Auf diese Weise entsteht ein kultureller Reproduktionskreislauf, der einerseits Prognosen hinsichtlich des Rückgriffs auf bestimmte Symboliken in dieser oder jener Situation, bei diesem oder jenem Ereignis erlaubt, der umgekehrt aber auch die Möglichkeit zur Intervention in Diskurse eröffnet, zum strategischen Einsatz von Kollektivsymbolen und zum Entwurf komplexer alternativer, kritischer oder gar gegen-hegemonialer Interdiskurse.

32 | Vgl. dazu Jürgen Link: Neue lange Märsche durch den Interdiskurs. Zu Neuerscheinungen von Jacques Ranciere, Renate Lachmann, Manfred Schneider, Rolf Kloepfer, Hanne Landbeck und Siegfried Jäger. In: kultuRRevolution. zeitschrift für angewandte diskurstheorie 28 (April 1993), S. 80-86, bes. 82f. (»Intertextualität und Interdiskurs [Renate Lachmann]").

33 | Uwe Wirth: Art. "Intermedialität». In: Roesler/Stiegler, Grundbegriffe (Anm. 16), S. 114-121, hier: S. 115. 


\section{DuEPublico}

Duisburg-Essen Publications online
UNIVERSITĀT

DE USS ISBN R G

offen im Denken

Ub $\begin{aligned} & \text { universitäts } \\ & \text { bibliothek }\end{aligned}$

Dieser Text wird über DuEPublico, dem Dokumenten- und Publikationsserver der Universität Duisburg-Essen, zur Verfügung gestellt. Die hier veröffentlichte Version der E-Publikation kann von einer eventuell ebenfalls veröffentlichten Verlagsversion abweichen.

DOI: $\quad 10.14361 /$ transcript.9783839407790.23

URN: urn:nbn:de:hbz:464-20210312-130047-2

Parr, Rolf. "Medialität und Interdiskursivität".

DOI: https://doi.org/10.14361/transcript.9783839407790.23

In: Medien des Wissens, edited by Georg Mein and Heinz Sieburg, Bielefeld: transcriptVerlag, 2011, eISBN: 9783839407790.

DOI: https://doi.org/10.14361/transcript.9783839407790, pp. 23-42.

(C) 2011 transcript Verlag, Bielefeld. Alle Rechte vorbehalten. 